Proceedings

\title{
Efficacy of Density in Predicting the Protective Properties of Padded Clothing in Rugby ${ }^{\dagger}$
}

\author{
Syed Imam 1,* , Heather Driscoll ${ }^{2}$, Keith Winwood ${ }^{3}$, Prabhuraj Venkatraman ${ }^{4}$ and Tom Allen ${ }^{1}$ \\ 1 Department of Engineering, Manchester Metropolitan University, Manchester M1 5GD, UK; \\ T.Allen@mmu.ac.uk \\ 2 Advanced Manufacturing Research Centre (AMRC), University of Sheffield, Sheffield S60 5BL, UK; \\ H.Driscoll@amrc.co.uk \\ 3 Department of Life Sciences, Manchester Metropolitan University, Manchester M1 5GD, UK; \\ K.Winwood@mmu.ac.uk \\ 4 School of Fashion, Faculty of Arts and Humanities, Manchester Metropolitan University, \\ Manchester M15 6BH, UK; P.Venkatraman@mmu.ac.uk \\ * Correspondence: Syed.A.Imam2@stu.mmu.ac.uk; Tel.: +44-759-845-7659 \\ + Presented at the 13th Conference of the International Sports Engineering Association, Online, \\ 22-26 June 2020.
}

Published: 15 June 2020

\begin{abstract}
World Rugby ${ }^{\mathrm{TM}}$ permits players to wear padded clothing meeting the requirements of Regulation-12, which limits density, thickness and impact performance. Due to non-uniform geometry, measuring the density of padding can be challenging and provides limited information on mechanical properties. This study investigated how well density could predict the impact performance of rugby padding, whilst reviewing compliance parameters as alternatives. Eleven samples of rugby padding, plus one control material, were tested for compliance, then impacted at energies of 4.9, 9.8 and $14.7 \mathrm{~J}$ using the setup as defined in Regulation-12, and finally cut to calculate density. The density and compliance parameters were correlated against peak impact accelerations using a Spearman's rank test. Density was not significantly correlated with peak acceleration at any energy level, with compliance tests significantly correlated with impact acceleration at only $4.9 \mathrm{~J}$.
\end{abstract}

Keywords: padded clothing; Regulation 12; density; compliance; World Rugby ${ }^{\mathrm{TM}}$

\section{Introduction}

Rugby has grown in popularity with participation rates increasing worldwide from 7.73 to 8.5 million within 2016 [1] and has high global economic impact [2]. With the plans of World Rugby ${ }^{\mathrm{TM}}$ to expand and involve more players [3], the market for rugby personal protective equipment (PPE) is likely to increase [4]. Sinclair [4] states that while there are scientific studies on the effectiveness of rugby PPE, both to ensure the protection of players and to substantiate manufacturers' claims, much controversy remains on its ability to reduce injury risk.

To govern the equipment used, World Rugby ${ }^{\mathrm{TM}}$ have defined a set of specifications and directives in their Handbook, specifically Regulation-12 Schedule 1: Specifications relating to Players' Dress [5]. Regulation-12 concentrates on player clothing, providing, (i) design and construction regulations; (ii) material specifications, and (iii) performance requirements and test methods. Under these sections, Regulation-12 specifies limitations on padding thickness $(10(+2) \mathrm{mm})$, material density $\left(45(+15) \mathrm{kgm}^{-3}\right.$ ) and impact performance (acceleration $>150 \mathrm{~g}$ for a $14.7 \mathrm{~J}$ impact, referred to as impact attenuation performance in Regulation-12).

Regulation-12 does not specify a method for measuring padding density. Padding often combines foam with fabric layers, typically compressed into regular or irregular geometries (Figure $1 \mathrm{a}-\mathrm{c})$. Density measurements according to ISO:845 [6] require foam samples to be cut into a shape 
such that its volume can be easily calculated. The combination of fabric and foam (typically bonded together) and the varied geometry of padding means density measurements can be challenging, and prone to uncertainty.

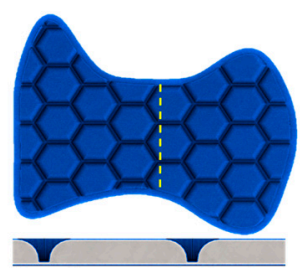

(a)

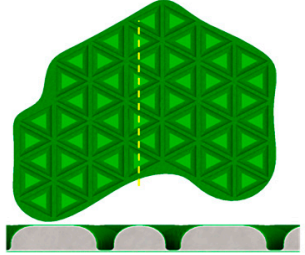

(b)

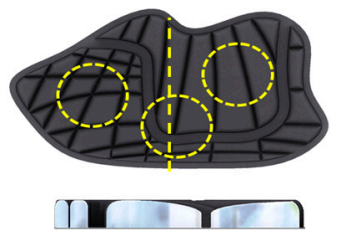

(c)

Figure 1. Computer-aided design reconstructions of approved paddings showing (a) hexagonal, (b) triangular and (c) irregular patterns with enlarged cross-sectional view showing variation within geometry.

With advances in manufacturing, the density of padding material can be tailored to enhance impact performance, as with auxetic [7] and biomimetic [8] foams. Impact testing of shin guards has shown that adding material, such as a sock, can reduce impact force [9]. Measuring the compliance of rugby padding using mechanical test methods as in ISO-2439 [10], would most likely be easier and more accurate than measuring its density. With World Rugby's intention to limit impact protection provided by padding ( $>150 \mathrm{~g}$ for a $14.7 \mathrm{~J}$ impact), compliance testing could complement the impact test. Compliance testing could be performed at low strain rates and prevent the use of stiffer materials, such as plastic shells, which could pass the impact test but change the nature of the game and may put players at risk of other injures, like cuts, lacerations and abrasions. This study investigated the potential of compliance tests for replacing the density criterion of padding in World Rugby Regulation- 12 .

\section{Materials and Methods}

Eleven designs of World Rugby ${ }^{\mathrm{TM}}$ approved jerseys were obtained from five manufacturers. Intact padding samples (size range: $150 \times 120 \mathrm{~mm}$ to $280 \times 220 \mathrm{~mm}$ ) were taken from the shoulder region. A $220 \times 150 \mathrm{~mm}$ sample of control material (Aortha White Plastazote ${ }^{\circledR}$-LD-60, Algeos), similar to the foam used in PPE [9], and meeting the thickness and density (LD-60 corresponds to $60 \mathrm{kgm}^{-3}$ ) requirements of Regulation-12, was also tested alongside the padding samples for compliance, impact performance and density.

\subsection{Compliance Testing}

Compliance testing was carried out according to ISO-2439 [10], using a uniaxial testing machine (Hounsfield HK10S) with a $1 \mathrm{kN}$ load cell ( $0.5 \%$ or $5 \mathrm{~N}$ accuracy) and a $62 \mathrm{~mm}$ diameter indenter, at a compression rate of $100 \mathrm{~mm} / \mathrm{min}$. Sample thicknesses were measured using a height gauge (Dial Gauge, J.E. Baty \& Co., Burgess Hill, UK, accuracy: $0.01 \mathrm{~mm}$ ) and used to calculate compressive strain. Samples were subjected to the five testing methodologies in the standard:

- A: Force at $40 \%$ strain after a 30 s hold;

- B: Forces at 25\% (B-25), 40\% (B-40) and 65\% (B-65) strain, after a 30 s hold at each strain;

- C: Instantaneous force at $40 \%$ strain;

- D: Force at $20 \%$ compression after a 20 s hold;

- E: Ratio of forces at $25 \%$ and $65 \%$ strain during compression cycle.

For Methods A to D, a higher force reading corresponds to a higher compliance. Method $\mathrm{E}$ gives a compression deflection coefficient and only provides additional information about the load bearing properties of the material and cannot be linked directly to its compliance. 


\subsection{Impact Testing}

Samples were impacted at energies of 4.9, 9.8 and $14.7 \mathrm{~J}$ using a $5 \mathrm{~kg}$ mass (flat face, $\varnothing 130 \mathrm{~mm}$ ) dropped from $0.1,0.2$ and $0.3 \mathrm{~m}$ (Figure 2). The anvil (horizontal steel cylinder, $\varnothing 115 \mathrm{~mm}$ ) was fixed on four load cells (208C05-Force Sensor, PCB Piezotronics) with a sampling rate of $20 \mathrm{kHz}$ and connected to an oscilloscope (PicoScope ${ }^{\circledR}$, Version 6, Pico Technology) via two 3-Channel ICP ${ }^{\circledR}$ sensor signal conditioners (480B21, $\mathrm{PCB}{ }^{\circledR}$ ) to record impact force. To measure deformation, each impact was filmed with a High-Speed Video (HSV) Camera (Phantom Miro R111, Vision Research, Wayne, NJ, USA) with a resolution of $512 \times 320$ and a sample rate of $10 \mathrm{kHz}$ and $99.00 \mu$ s exposure rate. The camera and load cells were synchronized using the oscilloscope.

Each sample was impacted three times at each energy with at least one minute between impacts. The sample was moved between impacts to change the impact location (as per Regulation-12: $20 \mathrm{~mm}$ from the periphery and minimum $30 \mathrm{~mm}$ apart). The voltage readings from each load cell was converted to force using the calibration factors (range: 0.2214 to $0.2399 \mathrm{mVN}^{-1}$ ) provided by the manufacturer. Peak force was divided by the product of the drop mass $(5 \mathrm{~kg})$ and the gravitational constant $\left(9.81 \mathrm{~ms}^{-2}\right)$ to give acceleration in $\mathrm{g}$ (as per Regulation-12).

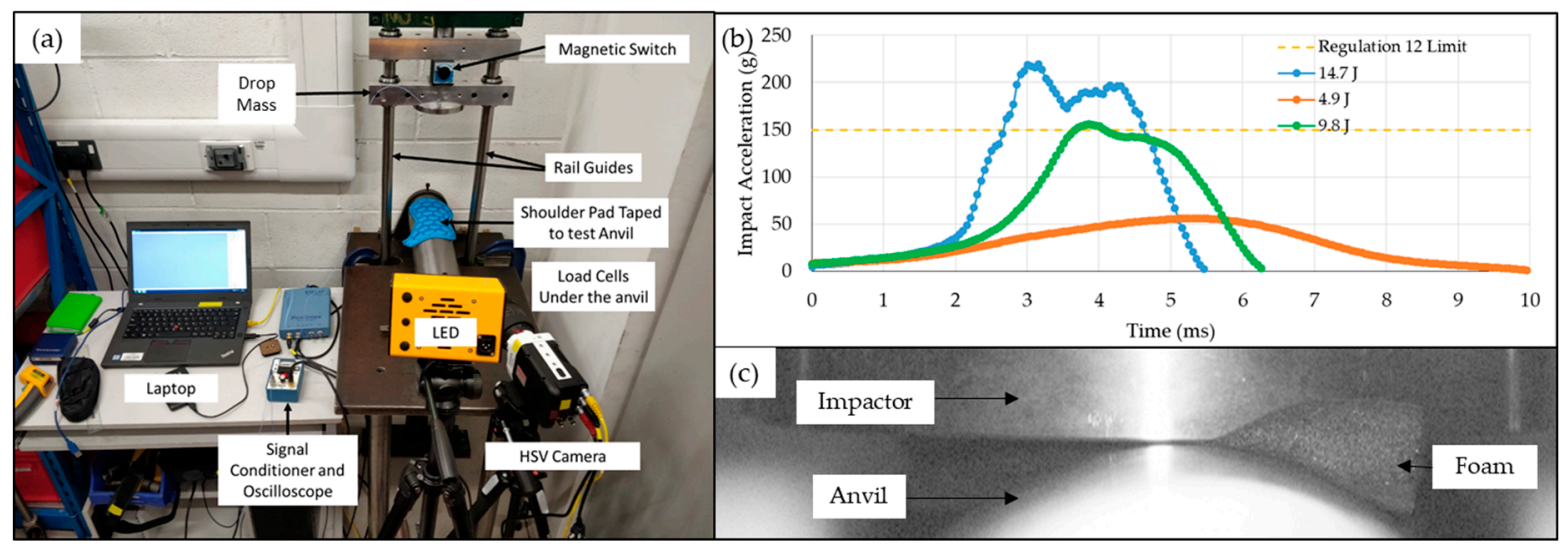

Figure 2. (a) Drop rig setup (as per Regulation-12) used for impact testing, (b) sample output graph Figure 4. 9, 9.8 and 14.7 J and (c) a High-Speed Video (HSV) image showing padding bottoming out at $4.9 \mathrm{~J}$.

\subsection{Density Testing}

Density measurements were carried out according to ISO:845 [9]. Following impact testing, five cylindrical specimens were cut from each padding sample (at varied locations) using a wad punch (Ø $9.8 \mathrm{~mm}$ to accommodate padding patterns). The diameter (mean of three locations: two ends and approximate middle) and height of each specimen were measured using a Vernier caliper (Composite Digital Vernier Caliper, Silverline ${ }^{\circledR}$ ) and used to calculate the volume. The mass of each cylindrical specimen was measured using a balance (ABS 220-4N, KERN ${ }^{\circledR}$, Germany). Density of the padding was calculated as the ratio of specimen mass to volume. The mean values for thickness, compliance, density and peak acceleration for each sample were compared using a Spearman's rank correlation at a confidence level of $95 \%$ using Minitab ${ }^{\circledR}$ (V18 Statistical software, USA).

The diameter and height of one cylindrical specimen was measured five times by three operators and the resultant density values were used to estimate the intra operator error $\left(E_{I N T R A}\right)$, as the coefficient of variation (CV) (Equation (1)).

$$
E_{\text {INTRA }}=\left(\frac{\sigma_{o p}}{\mu_{o p}}\right) \times 100 \%
$$

where $\sigma_{o p}$ is the standard deviation and $\mu_{\mathrm{op}}$ is the mean density for each operator.

Ten cylindrical specimens (5 from Sample 1 and 5 from Sample 6) were measured once by two additional operators (giving three total) to estimate inter-operator error $E_{I N T E R}$ (Equation (2)). 


$$
E_{\text {INTER }}=\sqrt{\left(\sigma_{1}+\sigma_{6}\right)}
$$

where $\sigma_{1}$ and $\sigma_{6}$ are the $\mathrm{CV}$ values for the two sets of five specimens (from Sample 1 and 6) for combined density measurements for all three operators (Equation (3)).

$$
\sigma_{n}=\left(\frac{\sigma_{\text {sample } n}}{\mu_{\text {sample } n} n}\right) \times 100,(n=1,6)
$$

where $\sigma_{\text {sample } n} n$ is the standard deviation and $\mu_{\text {sample_n}} n$ is the mean of all density measurements for a sample set.

The error propagation $\left(E_{P R O P}\right)$ during the density calculation was estimated using the accuracy of the measuring instruments for variables in the density equation (Equation (4)) [11].

$$
E_{P R O P}=\sqrt{\left(\frac{A_{B}}{m}\right)^{2}+2\left(\frac{A_{C}}{d}\right)^{2}+\left(\frac{A_{C}}{h}\right)^{2}}
$$

where $A_{B}$ is balance accuracy $(0.1 \mathrm{mg}), \mathrm{m}$ is the measured mass, $A_{c}$ is the caliper accuracy $(0.01 \mathrm{~mm})$, $d$ is the measured diameter and $h$ is the measured height.

\section{Results}

Force values increased with applied strain (B-65 > B-40 > B-25), while instantaneous readings were typically higher than readings after a hold period $(C>A)$ (Table 1$)$. Some density values exceeded the Regulation-12 limit. For all samples, peak acceleration increased with impact energy (Table 2). All compliance methods (apart from E, which was positive) and thickness had significant negative correlation with peak impact acceleration at $4.9 \mathrm{~J}$ (Table 3), meaning peak acceleration decreased as sample compliance or thickness increased. Density was not significantly correlated with

\begin{tabular}{|c|c|c|c|c|c|c|c|c|c|}
\hline \multirow{2}{*}{ S. No. } & \multirow{2}{*}{$\begin{array}{c}\text { Thickness } \\
\text { (mm) }\end{array}$} & \multicolumn{7}{|c|}{ Force Values (N) } & \multirow{2}{*}{$\begin{array}{l}\text { Density } \\
\left(\mathrm{kgm}^{-3}\right)\end{array}$} \\
\hline & & $\mathbf{A}$ & B-25 & B-40 & B-65 & $\mathrm{C}$ & $\mathrm{D}$ & $\mathrm{E}^{\mathrm{A}}$ & \\
\hline 1 & $10.3 \pm 0.2$ & $220 \pm 3$ & $129 \pm 2$ & $234 \pm 1$ & $590 \pm 13$ & $248 \pm 1$ & $131 \pm 7$ & $4.3 \pm 0.1$ & $41.1 \pm 0.8$ \\
\hline 2 & $10.5 \pm 0.3$ & $178 \pm 2$ & $97 \pm 4$ & $187 \pm 8$ & $511 \pm 31$ & $175 \pm 10$ & $102 \pm 2$ & $5.0 \pm 0.2$ & $71.5 \pm 1.0^{\text {в }}$ \\
\hline 3 & $9.6 \pm 0.2$ & $154 \pm 10$ & $90 \pm 4$ & $171 \pm 9$ & $460 \pm 36$ & $184 \pm 10$ & $83 \pm 5$ & $4.9 \pm 0.3$ & $72.4 \pm 1.3^{\text {в }}$ \\
\hline 4 & $10.2 \pm 0.1$ & $204 \pm 2$ & $111 \pm 6$ & $206 \pm 9$ & $518 \pm 27$ & $236 \pm 4$ & $118 \pm 1$ & $4.4 \pm 0.2$ & $40.2 \pm 1.6$ \\
\hline 5 & $9.8 \pm 0.1$ & $96 \pm 19$ & $45 \pm 6$ & $124 \pm 5$ & $330 \pm 49$ & $201 \pm 6$ & $33 \pm 4$ & $5.9 \pm 0.7$ & $27.2 \pm 2.4$ \\
\hline 6 & $11.0 \pm 0.2$ & $145 \pm 7$ & $76 \pm 7$ & $156 \pm 6$ & $423 \pm 5$ & $162 \pm 8$ & $77 \pm 5$ & $4.9 \pm 0.2$ & $49.4 \pm 0.8$ \\
\hline 7 & $10.1 \pm 0.3$ & $169 \pm 11$ & $102 \pm 12$ & $187 \pm 13$ & $452 \pm 33$ & $189 \pm 10$ & $94 \pm 3$ & $3.9 \pm 0.1$ & $82.3 \pm 2.5^{\text {в }}$ \\
\hline 8 & $7.7 \pm 0.3$ & $97 \pm 9$ & $44 \pm 8$ & $104 \pm 14$ & $368 \pm 34$ & $105 \pm 12$ & $38 \pm 3$ & $7.2 \pm 0.1$ & $109.1 \pm 3.3^{\text {в }}$ \\
\hline 9 & $8.1 \pm 0.4$ & $128 \pm 4$ & $60 \pm 4$ & $134 \pm 4$ & $409 \pm 13$ & $112 \pm 7$ & $59 \pm 3$ & $5.3 \pm 0$ & $72.9 \pm 5.0^{\text {в }}$ \\
\hline 10 & $11.0 \pm 0.3$ & $196 \pm 41$ & $96 \pm 10$ & $197 \pm 18$ & $577 \pm 83$ & $207 \pm 38$ & $78 \pm 8$ & $4.8 \pm 0.3$ & $65.3 \pm 2.6^{\mathrm{B}}$ \\
\hline 11 & $5.8 \pm 0.1$ & $46 \pm 1$ & $33 \pm 1$ & $54 \pm 4$ & $174 \pm 22$ & $64 \pm 4$ & $24 \pm 3$ & $6.1 \pm 0.4$ & $259.5 \pm 4.7$ в \\
\hline $12^{\mathrm{C}}$ & $10.2 \pm 0.2$ & $274 \pm 14$ & $202 \pm 17$ & $354 \pm 19$ & $781 \pm 63$ & $337 \pm 16$ & $169 \pm 1$ & $2.9 \pm 0$ & $53.5 \pm 0.5$ \\
\hline
\end{tabular}
peak impact acceleration at any impact energy.

Table 1. Force measurements using the five different methods in ISO: 2439 and density. (mean \pm SD).

${ }^{\mathrm{A}}$ has no unit. ${ }^{\mathrm{B}}$ Density outside regulation-12 limits $\left(45+(15) \mathrm{kgm}^{-3}\right) .{ }^{\mathrm{C}}$ Control material.

Sample deformation, measured using the HSV showed $>90 \%$ compression for all samples at 9.8 and 14.7 J. When measuring samples, $E_{\text {INTRA }}$ values were $1.8 \%, 2.4 \%$ and $5.9 \%$ (mean error: $4.1 \%$ ), $E_{I N T E R}$ was $16.9 \%$ (value range sample 1: 40.3 to $52.2 \mathrm{kgm}^{-3}$, sample $6: 48.6$ to $68.0 \mathrm{kgm}^{-3}$ ), and $E_{P R O P}$ was $0.2 \%$.

The results from the correlation testing (Table 3), although not significant, indicate that changing the impact energy changed the relationship (positive to negative) between both peak impact acceleration and density and peak impact acceleration and compliance. Figure 3 illustrates how the relationship between compliance measured using Method $C$ and peak impact acceleration changed from negative to positive as impact energy increased. 
Table 2. Peak impact acceleration at three impact energies (mean \pm SD).

\begin{tabular}{cccc}
\hline \multirow{2}{*}{ S No. } & \multicolumn{3}{c}{ Peak Impact Acceleration $(\mathrm{g})$} \\
\cline { 2 - 4 } & $\mathbf{4 . 9} \mathbf{~ J}$ & $\mathbf{9 . 8} \mathbf{~ J}$ & $\mathbf{1 4 . 7} \mathbf{~}$ \\
\hline 1 & $57 \pm 1.4$ & $153 \pm 2.6$ & $220 \pm 1.2$ \\
2 & $57 \pm 1.5$ & $151 \pm 0.7$ & $221 \pm 0.7$ \\
3 & $71 \pm 2.1$ & $163 \pm 6.3$ & $229 \pm 1.1$ \\
4 & $62 \pm 0.1$ & $148 \pm 4.4$ & $223 \pm 2.9$ \\
5 & $106 \pm 4.5$ & $171 \pm 2.0$ & $232 \pm 1.3$ \\
6 & $66 \pm 2.3$ & $149 \pm 3.0$ & $223 \pm 1.4$ \\
7 & $56 \pm 2.3$ & $138 \pm 4.0$ & $210 \pm 5.2$ \\
8 & $113 \pm 2.6$ & $181 \pm 4.4$ & $222 \pm 2.3$ \\
9 & $91 \pm 4.2$ & $174 \pm 2.5$ & $220 \pm 4.4$ \\
10 & $70 \pm 7.9$ & $156 \pm 6.5$ & $215 \pm 7.5$ \\
11 & $84 \pm 0.8$ & $146 \pm 0.6$ & $183 \pm 1.3$ \\
$12^{\mathrm{C}}$ & $44 \pm 0.7$ & $95 \pm 4.2$ & $155 \pm 5.7$ \\
\hline
\end{tabular}

Table 3. Spearman's (rho) Correlation Matrix.

\begin{tabular}{ccccc}
\hline & 4.9 J & $\mathbf{9 . 8} \mathrm{J}$ & $\mathbf{1 4 . 7} \mathrm{J}$ & Density \\
\hline Thickness & -0.593 * & -0.274 & -0.014 & - \\
Density & 0.224 & 0.042 & -0.434 & - \\
A & $-0.804 *$ & -0.434 & -0.294 & -0.441 \\
B-25 & $-0.881 *$ & -0.531 & -0.280 & -0.455 \\
B-40 & $-0.816^{*}$ & -0.462 & -0.280 & -0.501 \\
B-65 & $-0.748^{*}$ & -0.357 & -0.280 & -0.427 \\
C & $-0.601 *$ & -0.336 & 0.098 & $-0.713 *$ \\
D & $-0.860 *$ & -0.483 & -0.231 & -0.392 \\
E-CDC & $0.862 *$ & $0.630 *$ & 0.371 & 0.378 \\
\hline * indicates significant correlation $p<0.05$.
\end{tabular}

The control material was less compliant than the rugby padding samples ( 100 N higher, using Method C) and had lower peak acceleration at all energy levels. At $14.7 \mathrm{~J}$, the mean impact acceleration of the control material exceeded the Regulation- 12 limit by $5 \mathrm{~g}$. The linear relationship between compliance (Method C) and peak impact acceleration at $14.7 \mathrm{~J}$ is skewed due to the lower peak impact acceleration of the control sample, although this is accounted for in Spearman's correlation (which gave a positive correlation).

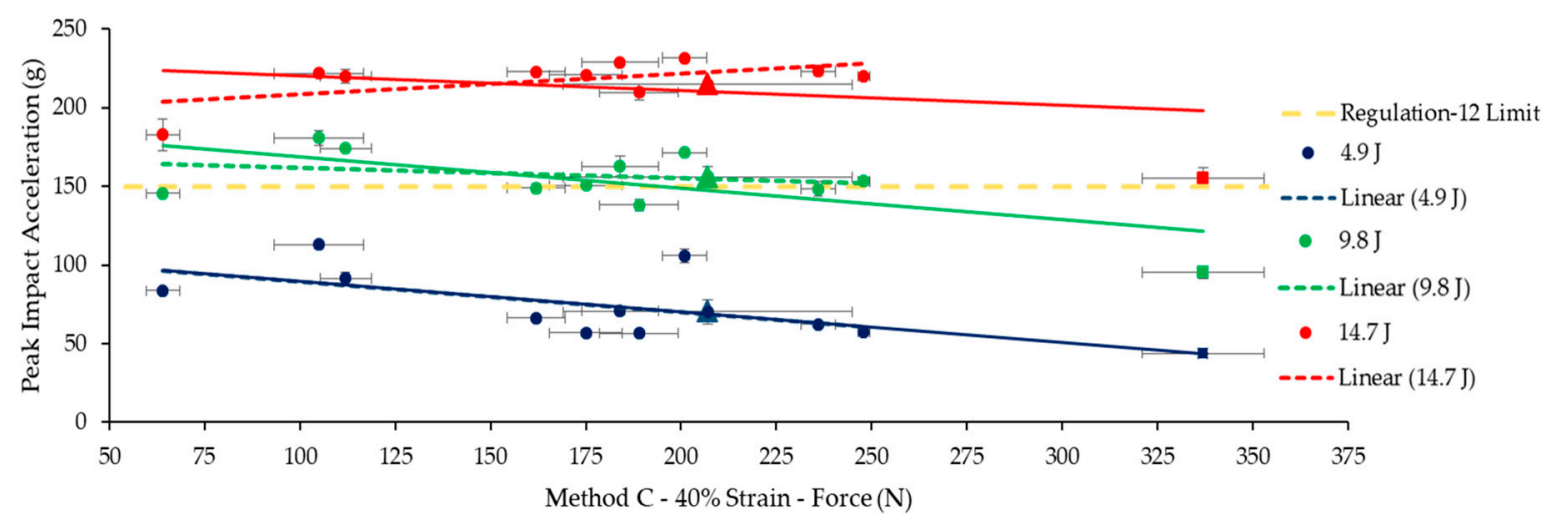

Figure 3. Peak impact acceleration vs. compliance at impact energies of 4.9, 9.8 and 14.7 J. Compliance measured using ISO-2439 Method C. The error bars indicate SD. Sample 10 (with high variance in compliance) is represented by triangles. The linear trend for padding excluding (dashed) and including (continuous) the control material (square) is shown. 


\section{Discussion}

All compliance test methods provided a statistically significant correlation $(p<0.05)$ to the peak impact acceleration at $4.9 \mathrm{~J}$ impact energy only, whereas padding density had no significant correlation at any energy level. Relationships changed between density and impact peak acceleration (positive to negative), thickness or compliance and peak impact acceleration (large negative to low negative/positive) as impact energy increased. At the lowest impact energy (4.9 J), more compliant foams gave higher peak acceleration on impact, while stiffer foams tended to withstand the impact and reduce peak acceleration. In contrast, for higher energy impacts, there was no significant correlation (apart from E at $9.8 \mathrm{~J}$ ) because the samples were bottoming out (compression $>90 \%$ ) and providing little to no resistance to impact as seen on the HSV image in Figure 2c (noted in Samples 1 to 11 at $9.8 \mathrm{~J}$ and in all samples at $14.7 \mathrm{~J}$ impact).

Large variances were noticed in compliance and peak impact values for Sample 10, which may be due to the irregular geometry highlighted in Figure 1c (Sample 10). While measuring density, it was noticed that the extracted cylindrical specimens were often warped. While the $E_{P R O P}$ during density calculations accounted for only $0.2 \%$ of the error, the $E_{I N T E R}$ was $17 \%$, indicating that measuring foam density can also be prone to human error. The presence of fabric in certain samples (as it was glued to the padding) added further uncertainty to the measured density values. These limitations provide some justification to replace the density criterion in Regulation- 12 with a compliance parameter that is easier to measure, although further work is needed.

When comparing the compliance testing methodologies, Methods A, B and D measure force after a period of hold. The often viscoelastic nature of padding material means that the stress can relax during this period of hold, with reducing force readings compared to the value at the end of the loading ramp. As stated in ISO:2439, the reduction in force values can be noticed when comparing values obtained from Methods A and C, with the instantaneous force values obtained using Method $\mathrm{C}$ being higher (or similar) for most samples.

Compliance testing can be applied to intact samples of padding (as they would be worn by rugby players), unlike density, which required the extraction of specimens. With $E_{\text {INTRA }}$ and $E_{\text {INTER }}$ equaling $4 \%$ and $17 \%$ respectively, measuring the density of a padding is error prone and does not appear to be a good indicator of impact performance. To improve our understanding of compliance parameters, padding materials with different density and compliance ranges, such those used in sports like ice hockey and American football, could be explored. Further research is to investigate the inter-user variances for compliance testing and inter-laboratory testing to gauge repeatability.

\section{Conclusions}

The compliance and densities of eleven samples of padded rugby clothing and one control material were compared against their peak acceleration for an impact test at 4.9, 9.8 and $14.7 \mathrm{~J}$. Compliance parameters and thickness showed a significant negative correlation with peak impact acceleration at only $4.9 \mathrm{~J}$, while density showed no significant correlation. Results at 9.8 and $14.7 \mathrm{~J}$ indicate that the padding materials bottomed out (as seen in HSV images) due to the rigid impactor and anvil. Compliance has shown potential to replace the density criterion in Regulation-12. Furthermore, quasi-static compliance testing could complement the impact testing methodology and prevent the use of stiff materials, such as stiff plastic shells, which may increase the risk of injuries such as cuts, lacerations and abrasions.

Funding: This research was funded by World Rugby and Manchester Metropolitan University.

Acknowledgments: We would like to thank World Rugby ${ }^{\mathrm{TM}}$ for funding this research. The manufacturers for providing samples. In addition the technicians: Stephen Moyle, Bob Bamford \& Anthony Dickenson for fabricating and developing the drop rig and Michael Green for assistance during testing.

Conflicts of Interest: The authors declare no conflict of interest. The results are for research purposes only and do not affect the approval status of the padding. 


\section{References}

1. Player Numbers-2017. World Rugby. Available online: https://www.worldrugby.org/development/playernumbers (accessed on 19 September 2019).

2. Chadwick, S.; Semens, A.; Schwarz, E.C.; Zhang, D. Economic Impact Report on Global Rugby Part III: Strategic and Emerging Markets; Centre for International Business of Sport, Coventry University: Coventry, UK, 2010.

3. World Rugby Press Release. 2016. Available online: https://www.world.rugby/news/203177?lang=en (accessed on 10 October 2019).

4. Sinclair, M. Protective Equipment in Rugby Literature Review. 2009. Available online: https://www.springboks.rugby/en/pages//media/project/sa\%20rugby/pagecontent/boksmart/medical\%20 protocol/protective\%20equipment $\% 20 \mathrm{in} \% 20 \mathrm{rugby} /$ review $\% 20 \% 20$ protective $\% 20$ equipment $\% 20 \mathrm{in} \% 20 \mathrm{rug}$ by\%20union (accessed on 28 September 2017).

5. Regulation 12: Provisions Relating to Players Dress, World Rugby Handbook; World Rugby: Dublin, Ireland, 2015; pp. 191-222.

6. International Organization for Standardization. ISO 845: Cellular Plastics and Rubbers-Determination of Apparent Density; International Organization for Standardization: Geneva, Switzerland, 2006.

7. Duncan, O.; Shepherd, T.; Moroney, C.; Foster, L.; Venkatraman, P.; Winwood, K.; Allen, T.; Alderson, A. Review of auxetic materials for sports applications: Expanding options in comfort and protection. Appl. Sci. 2018, 8, 941.

8. Svagan, A.J.; Samir, M.A.A.; Berglund, L.A. Biomimetic foams of high mechanical performance based on nanostructured cell walls reinforced by native cellulose nanofibrils. Adv. Mater. 2008, 20, 1263-1269.

9. Ruznan, W.S.; Laing, R.M.; Lowe, B.J.; Wilson, C.A. Impact attenuation provided by shin guards for field hockey. Sports Eng. 2018, 21, 161-175.

10. International Organization for Standardization. ISO 2439: Flexible Cellular Polymeric MaterialsDetermination of Hardness (Indentation Technique); International Organization for Standardization: Geneva, Switzerland, 2008.

11. A Summary of Error Propagation. Instructional Physics Laboratory, Harvard University. 2013. Available online: http://ipl.physics.harvard.edu/wp-uploads/2013/03/PS3_Error_Propagation_sp13.pdf.(accessed on 21 September 2019).

(C) 2020 by the authors. Licensee MDPI, Basel, Switzerland. This article is an open access article distributed under the terms and conditions of the Creative Commons Attribution (CC BY) license (http://creativecommons.org/licenses/by/4.0/). 